\title{
DIAGNÓSTICOS DE ENFERMAGEM RELACIONADOS AO FENÔMENO AMAMENTAÇÃO EXCLUSIVA
}

Cândida Caniçali Primo¹, Luyanne Azevedo Cabral², Sarah Colombi Cuquetto², Mariana Rabello Laignier², Eliane de Fátima Almeida Lima ${ }^{3}$, Franciéle Marabotti Costa Leite ${ }^{4}$

\begin{abstract}
RESUMO: Na assistência à mulher em amamentação percebe-se a necessidade de aperfeiçoamento e individualização do cuidado de enfermagem e a utilização de terminologia padronizada que favoreça a troca de informação e a uniformização da comunicação entre os enfermeiros. Trata-se de um estudo exploratório-descritivo desenvolvido com o objetivo de construir afirmativas de diagnósticos /resultados para o fenômeno Amamentação exclusiva, tendo como base as diretrizes do Conselho Internacional de Enfermeiras e os termos do Modelo de Sete Eixos da Classificação Internacional para a Prática de Enfermagem (CIPE®) Versão 1.0, complementados com os da literatura da área. As combinações de termos resultaram em seis diagnósticos/resultados de enfermagem. Conclui-se que a CIPE® pode ser um facilitador na implantação do processo de enfermagem, dando suporte à documentação sistemática das atividades e proporcionando o emprego de terminologia específica, o que contribui para organizar a assistência às mulheres em amamentação.
\end{abstract}

DESCRITORES: Aleitamento materno; Diagnóstico de enfermagem; Terminologia; Classificação; Processos de enfermagem.

\section{NURSING DIAGNOSES RELATED TO THE PHENOMENON OF EXCLUSIVE BREASTFEEDING}

ABSTRACT: In caring for the breast-feeding woman, one can perceive the need for improvement and individualization of the nursing care and the use of standardized terminology, which favors the exchange of knowledge and the uniformization of communication between nurses. This is an exploratory-descriptive study undertaken with the objective of constructing assertions of diagnoses/results for the phenomenon of Exclusive breastfeeding, based on the guidelines of the International Council of Nurses and the terms of the Seven Axis Model of the International Classification for Nursing Practice (ICNP $\left.{ }^{\circledR}\right)$ Version 1.0, complemented with those of the literature in the area. The combinations of terms resulted in six nursing diagnoses/results. It is concluded that the CIPE® can be facilitative in the implantation of the nursing process, supporting the systematic documentation of the activities and making possible the use of specific terminology, which contributes to organizing the assistance to breast-feeding women.

DESCRIPTORS: Maternal breastfeeding; Nursing diagnosis; Terminology; Classification; Nursing processes.

\section{DIAGNÓSTICOS DE ENFERMERÍA RELACIONADOS AL FENÓMENO AMAMANTAMIENTO EXCLUSIVO}

RESUMEN: Acerca de la asistencia a la mujer en amamantamiento, se puede percibir la necesidad de perfeccionamiento e individualización del cuidado de enfermería y la utilización de terminología tipificada que favorezca el cambio de información y la tipificación de la comunicación entre los enfermeros. Es un estudio exploratorio y descriptivo desarrollado con el objetivo de construir afirmativas de diagnósticos/resultados para el fenómeno Amamantamiento exclusivo, con base en las directrices del Consejo Internacional de Enfermeras y los términos del Modelo de Siete Ejes de la Clasificación Internacional para la Práctica de Enfermería (CIPE®) Versión 1.0, complementados con los de la literatura del área. Las combinaciones de términos resultaron en seis diagnósticos/resultados de enfermería. Se concluye que CIPE® puede ser un facilitador en la implantación del proceso de enfermería, dando apoyo a la documentación sistemática de las actividades y proporcionando el empleo de terminología específica, lo que contribuye para organizar la asistencia a las mujeres en amamantamiento.

DESCRIPTORES: Amamantamiento materno; Diagnóstico de enfermería; Terminología; Clasificación; Procesos de enfermería.

${ }^{1}$ Enfermeira. Mestre em Saúde Coletiva. Professora do Curso de Graduação e do Mestrado Profissional em Enfermagem da Universidade Federal do Espírito Santo - UFES. Membro do Grupo CUIDAR: Ensino e Pesquisa em Enfermagem.

${ }^{2}$ Enfermeira.

${ }^{3}$ Enfermeira. Mestre em Enfermagem. Professora do Curso de Graduação em Enfermagem da UFES. Membro do Grupo CUIDAR: Ensino e Pesquisa em Enfermagem.

${ }^{4}$ Enfermeira. Mestre em Saúde Coletiva. Professora do Curso de Graduação em Enfermagem da UFES. Membro do Grupo CUIDAR: Ensino e Pesquisa em Enfermagem.

Autor correspondente:

Recebido: $13 / 08 / 2012$

Cândida Caniçali Primo Aprovado: 18/03/2013

Universidade Federal do Espírito Santo

Rua João de Oliveira Soares, 241 - 29090-390 - Vitória-ES-Brasil

E-mail: candidaprimo@gmail.com 


\section{INTRODUÇÃO}

O aleitamento materno representa a forma natural de nutrição do ser humano nos primeiros meses de vida. A aderência da puérpera à amamentação exclusiva e o contato precoce traz benefícios para o binômio mãe-filho, para a família, para a equipe prestadora do cuidado e para a sociedade ${ }^{(1-2)}$. Dentre as vantagens, as mulheres em amamentação exclusiva têm menor incidência de anemia, por diminuir o sangramento pós-parto; contracepção por seis meses; recuperação rápida do peso anterior a gestação; fator de proteção para câncer de mama, ovário e endométrio e

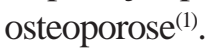

Além dos benefícios para a mãe, o aleitamento materno exclusivo para os lactentes proporciona maior proteção contra o risco de asma, o surgimento de diabetes mellitus tipo 1 e infecções ${ }^{(3)}$. A amamentação exclusiva protege o recém-nascido durante a vigência do aleitamento, através de uma proteção passiva, e diminui a taxa de mortalidade infantil, reduzindo o risco de diarreias agudas e persistentes, septicemia neonatal, alergias e outros agravos ${ }^{(4)}$.

As vantagens do aleitamento materno exclusivo para a sociedade, família e instituição de saúde, incluem a diminuição do lixo inorgânico, economia com alimentação do recém-nascido, consultas médicas, exames laboratoriais, hospitalização da criança; redução de gastos na aquisição de fórmulas, frascos, bicos e medicamentos (ocitocina); otimização da equipe profissional com a promoção do alojamento conjunto e retirada do berçário de recém-nascidos normais ${ }^{(1,4)}$.

Enfermeiros de alojamento conjunto e atuantes em maternidades, juntamente com a equipe multidisciplinar, devem estar atentos às reais necessidades da puérpera e do recém-nascido com foco na relação humana entre profissional e paciente, na educação e na orientação à saúde para que as mulheres adquiram segurança e tranquilidade ao assumir seu papel de mãe ${ }^{(5)}$. $O$ atendimento prestado deve ser humanizado mediante a utilização de conhecimentos técnicos e científicos e dos recursos disponíveis à realidade das mães e da instituição $0^{(1)}$.

Para facilitar o atendimento do enfermeiro torna-se necessário o uso de linguagem específica ou terminologia, na qual o princípio fundamental é a pertinência dos termos a áreas temáticas, estruturadas por meio de sistemas de classificação de saberes específicos. A proposição da pesquisa terminológica é identificar termos que expressam conhecimentos especializados sendo a sua função principal disseminar e autenticar o uso terminológico ${ }^{(6)}$.

Terminologia é compreendida como uma área de conhecimentos e práticas, sendo os termos técnico-cien- tíficos o principal objeto de estudos teóricos e aplicados, constituindo e sendo utilizadas no ambiente de atividades profissionais especializadas, veiculando conceitos próprios de cada área do conhecimento ${ }^{(7)}$. Na sua função de representação, éútil para três tipos de disciplinas ou atividades: a documentação, a linguística computacional e as especialidades basicamente técnicas-científicas. Englobando essas três vertentes, a terminologia se aplica para compor um objeto de trabalho próprio e para organizar de maneira mais adequada o processo terminológico, através de suas aplicações. É peça chave na representação do conteúdo dos documentos e para constatá-los ${ }^{(8)}$.

Dessa forma, o Conselho Internacional de Enfermagem (CIE), buscando estabelecer uma classificação que representasse a Enfermagem em âmbito mundial, votou e aprovou o desenvolvimento de um sistema de Classificação Internacional para a Prática de Enfermagem (CIPE®), no Congresso Quadrienal do CIE, sediado em Seul no ano de $1989^{(9)}$.

O primeiro passo para elaboração da CIPE® foi a realização de um levantamento em todo o mundo sobre os sistemas de classificação utilizados pela Enfermagem, através de pesquisas na literatura específica e pesquisas vinculadas às organizações membros do $\mathrm{CIE}^{(10)}$. Após o primeiro passo, com a intenção de identificar denominações inerentes à Enfermagem, foram analisados 14 sistemas de classificação, a Classificação Internacional de Doenças CID10, a Classificação Internacional de Funcionalidades, Incapacidades e Saúde e outras classificações aprovadas pela Organização Mundial da Saúde (OMS) ${ }^{(11-12)}$.

Em 1993, o CIE lançou um documento com o título Nursing's Next Advance: the International Classification for Nursing Practice- ICNP (Próximo Avanço da Enfermagem: uma Classificação Internacional para a Prática de Enfermagem-CIPE®), que continha, em ordem alfabética, os elementos da prática de enfermagem: os diagnósticos e os resultados de enfermagem ${ }^{(13)}$. Três anos depois o CIE publicou a Classificação Internacional para a Prática de Enfermagem- Versão Alfa, considerada um marco unificador contendo a classificação de fenômenos e de ações de enfermagem $^{(14)}$.

Em 1999 foi publicada a CIPE® Versão Beta, que possui uma estrutura multiaxial, e passou por uma revisão sugerida por enfermeiros que trabalharam com essa versão. Dessa revisão foi publicada a CIPE® Versão Beta 2, que teve como principal alteração a gramática, correções ou alterações de códigos e correções das definições. Nessa Versão constavam 8 eixos na estrutura de classificação dos fenômenos e das ações de enfermagem ${ }^{(15)}$.

Em 2005, no XXIII Congresso Quadrienal do CIE foi lançada a CIPE® Versão 1.0. Trata- se de um modelo 
simplificado de 7 eixos, que utiliza vocabulários já existentes e podem gerar novos vocabulários utilizando uma terminologia composicional ${ }^{(16)}$. Em 2008, o CIE apresentou uma nova versão da CIPE®, a 1.1, da qual não havia previsão de versão impressa, sendo disponível apenas a versão na WEB. Nesta versão foram incluídas declarações de diagnósticos, resultados e intervenções de enfermagem no corpo da classificação. No final de 2008, a CIPE® foi incluída na Família de Classificações Internacionais da Organização Mundial de Saúde (FCI - OMS), tornando-se a terminologia padronizada que representa o domínio da prática e unifica a Enfermagem a nível mundial ${ }^{(17)}$. Um ano após, durante o XXIV Congresso do CIE na África do Sul, foi lançada a CIPE® Versão 2.0 que possui mais de 400 novos conceitos em sua estrutura, resultantes do trabalho coordenado pelo Conselho no sentido de garantir a consistência e precisão da classificação ${ }^{(17)}$.

A CIPE® subsidia a qualidade da assistência de enfermagem, sendo um dispositivo para documentação e descrição da prática dos enfermeiros, contribuindo para a qualidade da assistência ao cliente ${ }^{(9)}$.

Diante do exposto, esta pesquisa tem como objetivo construir, com base na Classificação Internacional para a Prática de Enfermagem (CIPE®) Versão 1.0, afirmativas de Diagnósticos/Resultados de Enfermagem para o Eixo/Foco "Amamentação exclusiva", tendo como base as diretrizes do Conselho Internacional de Enfermeiras e os termos do Modelo de Sete Eixos da CIPE® Versão 1.0, complementados com os da literatura da área.

\section{MÉTODO}

Trata-se de um estudo exploratório-descritivo, cuja realização foram percorridas duas etapas, conforme descrito a seguir:

\section{Revisão da literatura sobre Amamentação Exclusiva}

Revisou-se as questões sobre classificação, fisiologia e orientações no período pós-parto por meio de livros textos da área de Obstetrícia, Aleitamento Materno, manuais do Ministério da Saúde e da Organização Mundial de Saúde. Além disso, realizou-se uma revisão da literatura nas bases de dados: Literatura Latino-Americana em Ciências da Saúde (LILACS) e Base de Dados de Enfermagem (BDENF), com os descritores: aleitamento materno e diagnóstico de enfermagem, nos idiomas português e espanhol. Os objetivos da revisão de literatura foram: análise de conceito de amamentação exclusiva e identificação dos atributos críticos ou essenciais de amamentação exclusiva, istoé, palavras ou expressões que aparecem repetidamente na literatura, que mostram a essência do conceito ${ }^{(18)}$.

\section{Composição de afirmativas de diagnósticos/ resultados de enfermagem relacionados à Amamentação Exclusiva}

Utilizou se os termos do Modelo de Sete Eixos da CIPE® e da literatura da área. O CIE recomenda que, para compor as afirmativas de diagnósticos, intervenções e resultados de enfermagem, se use o Modelo de Sete Eixos da CIPE®. Para a construção de diagnósticos/resultados de enfermagem são apontadas as seguintes diretrizes: incluir, obrigatoriamente, um termo do eixo Foco (área de atenção que é relevante para a Enfermagem); e um termo do eixo Julgamento (opinião clínica ou determinação relacionada ao foco da prática); incluir termos adicionais dos outros eixos, conforme a necessidade ${ }^{(9)}$.

\section{RESULTADOS}

Utilizaram-se as definições conceituais apresentadas na CIPE®, acrescentando a definição de alguns termos, baseando-se na literatura, por não apresentar na CIPE® clareza no seu enunciado. Após pesquisa em dicionário da língua portuguesa e na literatura específica, considerou-se que Aleitamento Materno e Amamentação são sinônimos $^{(19)}$, cujos resultados estão apresentados no quadro 1.

Quadro 1 - Identificação dos usos do conceito Amamentação Exclusiva no âmbito da literatura. Vitória, 2012

\begin{tabular}{|l|l|}
\hline CIPE® versão 1.0 & $\begin{array}{l}\text { "[...] quando a criança recebe somente leite materno, direto da mama ou ordenhado, ou } \\
\text { leite humano de outra fonte, sem outros líquidos ou sólidos, com exceção de gotas ou } \\
\text { xaropes contendo vitaminas”(9:56). }\end{array}$ \\
\hline $\begin{array}{l}\text { Organização } \\
\text { Mundial de Saúde }\end{array}$ & $\begin{array}{l}\text { "[...] quando a criança recebe somente leite materno, direto da mama ou ordenhado, ou } \\
\text { leite humano de outra fonte, sem outros líquidos ou sólidos, com exceção de gotas ou } \\
\text { xaropes contendo vitaminas, minerais ou outros medicamentos”(20:17). }\end{array}$ \\
\hline $\begin{array}{l}\text { Dicionários de } \\
\text { língua portuguesa }\end{array}$ & Amamentar, dar de leite a, nutrisse, tomar alento a. Aleitação - aleitamento ${ }^{(19)}$. \\
\hline Dicionário Médico & Criar com leite. Amamentação. Palavra mais correta alactamento ${ }^{(21)}$. \\
\hline
\end{tabular}


Na revisão da literatura encontrou-se 16 artigos científicos relacionados aos descritores, no entanto, após a leitura verificou-se que 8 artigos estavam repetidos nas bases de dados. Quanto aos diagnósticos de enfermagem 7 artigos utilizavam a Taxonomia North American Nursing Diagnoses Association (NANDA) e identificaram-se os seguintes diagnósticos: Amamentação Eficaz; Amamentação Ineficaz; Amamentação Interrompida; Padrão Ineficaz de Alimentação do Lactente. Um artigo utilizava a CIPE® e encontrou o diagnóstico "Amamentação Adequada”.

Considerando que a CIPE ${ }^{\circledR} 1.0$ traz no Eixo Foco os termos "Amamentação exclusiva" e "Amamentação" como fenômenos distintos e esse estudo visa abordar o Foco “Amamentação exclusiva”, percebe-se que não se verificou estudos que abordassem esse fenômeno.

A análise da literatura permitiu identificar os atributos essenciais e a amamentação exclusiva é evidenciada quando a criança recebe somente leite materno, direto da mama, leite materno ordenhado, leite humano de outra fonte, não recebe outros líquidos ou sólidos, recebe gotas ou xaropes contendo vitaminas, minerais ou outros medicamentos.

Dentre os 34 termos do Eixo Julgamento su- geridos pela CIPE® 1.0 utilizou-se cinco deles: melhorado, comprometido, potencial para aumento, interrompido e risco.

Devido a algumas definições apresentarem pouca clareza na tradução da versão CIPE® 1.0 optou-se por utilizar outras palavras que possuem correlação com os julgamentos supracitados, e, que foram extraídos de dicionário da língua portuguesa ${ }^{(19)}$, bem como, da literatura específica da amamentação, na elaboração deste trabalho.

O termo "Melhorado" foi substituído por Eficaz, entendido como aquele que produz o efeito desejado, que dá bom resultado: medida eficaz; tratamento eficaz, que age com eficiência ${ }^{(19)}$. Para o termo "Comprometido" utilizou-se Ineficaz, dado como aquele que não é eficaz, inútil, impróprio, inconveniente ${ }^{(19)}$.

Os termos do Eixo Julgamento são de grande relevância na determinação clínica de um fenômeno de enfermagem, demonstrando o grau de comprometimento ou efetividade do que está ocorrendo ${ }^{(22)}$. Para definição dos termos do Eixo Julgamento, que não compõem a lista dos apresentados na CIPE ${ }^{\circledR}$ Versão 1.0, foram elaboraram as definições, tendo como referencial dicionário da língua portuguesa ${ }^{(19)}$. Os diagnósticos pré-definidos estão descritos no quadro 2.

Quadro 2 - Diagnósticos de enfermagem para amamentação exclusiva segundo a CIPE® 1.0. Vitória, 2012

\begin{tabular}{|c|c|}
\hline Diagnósticos para Amamentação Exclusiva & Definição proposta \\
\hline Amamentação Exclusiva Eficaz & $\begin{array}{l}\text { Alimentar exclusivamente com leite materno, excluindo } \\
\text { todos os outros alimentos, nos primeiros quatro a seis } \\
\text { meses de vida, produzindo o efeito desejado e o resultado } \\
\text { esperado }\end{array}$ \\
\hline Amamentação Exclusiva Ineficaz & $\begin{array}{l}\text { Alimentar exclusivamente com leite materno, excluindo } \\
\text { todos os outros alimentos, nos primeiros quatro a seis me- } \\
\text { ses de vida, porém, de maneira imprópria e não eficaz }{ }^{(9,19)} \text {. }\end{array}$ \\
\hline $\begin{array}{l}\text { Potencial para Aumento da Amamentação } \\
\text { Exclusiva }\end{array}$ & $\begin{array}{l}\text { Alimentar exclusivamente com leite materno, excluindo } \\
\text { todos os outros alimentos, com capacidade para melhorar, } \\
\text { crescer ou desenvolver sua vontade de amamentar nos } \\
\text { primeiros quatro a seis meses de vida }{ }^{(3,9,25)} \text {. }\end{array}$ \\
\hline Amamentação Exclusiva Interrompida & $\begin{array}{l}\text { Alimentar exclusivamente com leite materno, excluindo } \\
\text { todos os outros alimentos, nos primeiros quatro a seis } \\
\text { meses de vida é definitivamente descontinuado }{ }^{(9)} \text {. }\end{array}$ \\
\hline Risco para Amamentação Exclusiva Ineficaz & $\begin{array}{l}\text { Alimentar exclusivamente com leite materno, excluindo } \\
\text { todos os outros alimentos, nos primeiros quatro a seis } \\
\text { meses de vida, porém, de maneira imprópria e não eficaz, } \\
\text { tendo a possibilidade de ocorrer algum problema }{ }^{(9,19)} \text {. }\end{array}$ \\
\hline Risco para Amamentação Exclusiva Interrompida & $\begin{array}{l}\text { Quando há possibilidade de se tornar definitivamente } \\
\text { descontinuado o processo de alimentar exclusivamente } \\
\text { com leite materno, excluindo todos os outros alimentos, } \\
\text { nos primeiros quatro a seis meses de vida }{ }^{(9)} \text {. }\end{array}$ \\
\hline
\end{tabular}

Cogitare Enferm. 2013 Abr/Jun; 18(2):215-21 


\section{DISCUSSÃO}

Em relação ao diagnóstico de enfermagem "Amamentação Exclusiva Eficaz" para que as mães consigam amamentar exclusivamente e de forma eficaz, até os seis meses, a Organização Mundial da Saúde e o Fundo das Nações Unidas para a Infância recomendam: iniciar a amamentação nas primeiras horas de vida da criança; amamentação exclusiva, ou seja, o lactante recebe apenas leite materno, sem nenhum outro alimento ou líquido, nem mesmo água; que aconteça sob livre demanda e não uso mamadeiras nem chupetas ${ }^{(23)}$.

Além disso, para conseguir amamentação exclusiva eficaz, a mulher deve oferecer uma mama de cada vez com o objetivo de promover maior esvaziamento da glândula e permitir que o recém-nascido receba o leite mais rico em gorduras, obtido ao final da mamada. Que na próxima mamada deve se iniciar pela mama que não foi ofertada na mamada anterior ou àquela que foi oferecida por último. Ainda que a duração de cada mamada é variável, sendo que o intervalo entre as mamadas dependerá da fome da criança. Recomenda-se que a criança seja colocada em esquema de livre demanda e pelo tempo necessário para satisfazer suas necessidades, o que pode variar para cada criança. $\mathrm{O}$ local para amamentar deverá ser escolhido pela mãe, desde que seja confortável. É necessário o uso do sutiã para manter a mama sempre elevada, prevenindo possíveis acotovelamentos de ductos e a sua troca deve ser diária. A região mamilo-areolar deve ser sempre lubrificada com o próprio leite e realizar banho de sol nos mamilos, com o objetivo de aumentar a resistência da região mamilo-areolar ${ }^{(1,24)}$.

Nesse sentido, a mulher e os profissionais de saúde devem estar aptos ao manejo das técnicas de amamentação, que tem como base o posicionamento da mãe e do bebê, pega, sucção e deglutição efetivas ${ }^{(2,4)}$. Assim, a posição para a mãe amamentar depende de vários fatores, entre eles o tipo de parto e o dia de puerpério que se encontra, sendo importante que em qualquer posição a mulher esteja confortável e relaxada. O posicionamento da criança é determinante para uma amamentação efetiva e o que deve ser considerado, independente da posição que a criança estiver, é que o seu corpo deve manter-se sempre em um único eixo. A preensão correta da região mamilo-areolar é um aspecto fundamental para determinar a efetividade da amamentação, uma vez que mediante a pega correta, a criança consegue retirar o leite em quantidade adequada, sem causar danos ao tecido da região abocanhada. É importante ressaltar que a boca do lactente deve estar bem aberta para fazer uma preensão de toda ou quase toda a região mamilo-areolar, de forma que mamilo e aréola fiquem completa, ou quase completamente, introduzidos em sua boca. Após a preensão, nariz e queixo devem ficar encostados na mama. Os lábios superior e inferior da criança devem estar virados para fora. A sucção da mama e deglutição são fundamentais para a amamentação eficaz, pois, por meio da sucção se mantém o estímulo adequado à produção e ejeção láctea, o esvaziamento adequado das mamas e, consequentemente, a satisfação da criança em relação às suas necessidades nutricionais e afetivas ${ }^{(24-25)}$.

Por outro lado, a "Amamentação Exclusiva Ineficaz" ocorre quando a pega e a posição da mãe e/ou do bebê estão incorretas, por prematuridade, o bebê não ganha peso, resistência e dificuldade do recém nascido de adaptação à técnica de amamentação. Também pode ocorrer quando o reflexo de descida do leite, sucção e busca do recém-nascido estão prejudicados ou há baixa produção de leite ${ }^{(14,26)}$.

Outros autores trazem que amamentação pode estar ineficaz quando os bebês não conseguem pegar a aréola adequadamente, ou não conseguem manter a pega. Além disso, o bebê pode não abocanhar adequadamente a mama, por apresentarem-se tensas, ingurgitadas, ou os mamilos são invertidos ou planos. Alguns bebês resistem às tentativas de serem amamentados, algumas vezes, e isso pode estar associado ao uso de bicos artificiais ou chupetas, ou ainda à presença de dor quando o bebê é posicionado para mamar. O manejo da amamentação vai depender da dificuldade detectada ${ }^{(1,4,26)}$.

Entende-se por "Potencial para Aumento da Amamentação Exclusiva" quando a mãe apresenta produção de leite adequada e sente-se motivada a amamentar exclusivamente por pelo menos seis meses ${ }^{(3)}$. $\mathrm{O}$ apoio familiar e profissional é a base para o sucesso da amamentação. Ouvir a mulher sobre seus anseios, dúvidas e verificar as experiências anteriores faz parte do processo de aprendizado da amamentação, apoiando-a e aumentando sua autoconfiança. Conhecer os aspectos relacionados à prática da amamentação é fator fundamental no sentido de colaborar para que a mãe possa se sentir disposta e apta a amamentar de forma efetiva e tranquila e, nesse contexto, o profissional de saúde tem papel fundamental, fornecendo as orientações necessárias e adequadas para o êxito materno ${ }^{(2,24,27)}$.

"Amamentação Exclusiva Interrompida" é quando o processo de alimentar exclusivamente com leite materno, excluindo todos os outros alimentos, nos primeiros quatro a seis meses de vida, é definitivamente descontinua- 
$\mathrm{do}^{(9)}$. Esta poderá ocorrer quando os fatores que tornam a amamentação exclusiva ineficaz evoluem, em casos de mastite, fissura mamilar, ingurgitamento mamário, abcesso e algumas doenças ou fármacos incompatíveis com o aleitamento ${ }^{(3,26)}$. Entre essas doenças se destacam as infectocontagiosas, que podem acometer tanto a mãe quanto a criança. No entanto, quando uma nutriz apresenta sintomas de uma doença infectocontagiosa, geralmente já expôs seu filho ao agente patogênico. Nesse caso, a manutenção da amamentação deve ser avaliada como forma de proteger a criança ${ }^{(24)}$. Nas infecções causadas pelos retrovírus (vírus da imunodeficiência humana, os vírus T-linfotrópico humano tipo 1 e T-linfotrópico humano tipo 2) a transmissão através do leite humano é mais frequente, por isso a amamentação deve ser contra-indicada ${ }^{(28)}$.

Quanto aos medicamentos incompatíveis com a amamentação, são citados como contraindicações absolutas ou relativas ao aleitamento, por exemplo, os antineoplásicos e radiofármacos. Como essas informações sofrem frequentes atualizações, recomenda-se que previamente à prescrição de medicações à nutrizes, essas questões sejam consultadas ${ }^{(27-28)}$.

Conforme descrito, existem alguns problemas enfrentados pelas nutrizes durante o aleitamento materno que, se não forem precocemente identificados e tratados, podem ser importantes fatores de "Risco para Amamentação Exclusiva Ineficaz ou Interrompida". Os profissionais de saúde têm um papel importante na prevenção e no manejo dessas dificuldades. Assim, a avaliação da mamada é uma atividade básica de apoio à amamentação. É o momento que permite a prevenção de problemas nas mamas, e a correção de eventuais erros de postura e pega. É fundamental para prover à mãe o apoio necessário nestes primeiros momentos com o bebê $\hat{e}^{(1,4,24)}$.

Revisão sistemática da literatura sugere que o profissional de saúde, em especial o enfermeiro, perceba a importância da comunicação como instrumento do processo de trabalho em saúde, utilize o diagnóstico de enfermagem com o objetivo de realizar a intervenção adequada, evitando dúvidas, dificuldades e possíveis complicações e investindo em atividades que incentivem a manutenção do aleitamento materno, a fim de evitar o desmame precoce ${ }^{(29)}$.

\section{CONSIDERAÇÕES FINAIS}

Encontraram-se algumas dificuldades durante a construção desses diagnósticos, visto que alguns termos do Eixo Julgamento da CIPE ${ }^{\circ}$ Versão 1.0 apresentavam definições pouco explicativas. Dessa forma, alguns desses foram substituídos, baseados nos conceitos de dicionário da língua portuguesa e da literatura específica.

Com essa pesquisa espera-se contribuir para a universalização da linguagem utilizada pelos enfermeiros, já que o uso da nomenclatura CIPE® pode ser um facilitador na implantação do processo de enfermagem. Também, dando suporte à documentação sistemática das atividades, proporcionando terminologia específica, que contribui para disseminar a prática e os conceitos da enfermagem.

Este estudo pode colaborar na formação dos profissionais de enfermagem, pois articula a assistência e o ensino ao elaborar um conjunto de diagnósticos de enfermagem para sistematizar a prática clínica da amamentação, facilitando a compreensão dos acadêmicos acerca dos fenômenos envolvidos no processo de amamentar. Também contribui na pesquisa em enfermagem, uma vez que serve de estímulo para o desenvolvimento de novos estudos que visem a confirmação e validação desses diagnósticos e o desenvolvimento de intervenções voltadas as mulheres lactantes considerando, principalmente, a demanda específica de cuidados de enfermagem direcionados ao manejo da amamentação.

\section{REFERÊNCIAS}

1. Febrasgo: Federação Brasileira das Associações de Ginecologia e Obstetrícia. Aleitamento materno: manual de orientação. São Paulo: Ponto; 2006.

2. Almeida IS, Ribeiro IB, Rodrigues BMRD, Costa CCP, Freitas NS, Vargas EB. Amamentação para mães primíparas: perspectivas e intencionalidades do enfermeiro ao orientar. Cogitare enferm. 2010;15(1):19-25.

3. Secretaria de Atenção à Saúde (BR). Departamento de Atenção Básica. Saúde da criança: nutrição infantil: aleitamento materno e alimentação complementar. Brasília: Ministério da Saúde; 2009.

4. Carvalho MR, Tamez RN. Amamentação: bases científicas. 2a ed. Rio de Janeiro. Guanabara Koogan; 2005.

5. Soares AVN, Gaidzinski RR, Cirico MOV. Identificação das intervenções de enfermagem no sistema de alojamento conjunto. Rev Esc Enferm. USP 2010;44(2):308-17.

6. Pavel S, Nolet D. Manual de terminologia. Public works and government services: Canada; 2003. 
7. Krieger MG. Do ensino da terminologia para tradutores: diretrizes básicas. Periódicos UFSC. 2011. [acesso em 10 abr 2011] Disponível: http://www.periodicos.ufsc.br/ index.php/traducao/article/viewFile/6862/6413

8. Cabré MT. La terminologia hoy, concepciones, tendências y aplicaciones. Cienc. Inf. 1995;(24)3:12.

9. Conselho Internacional de Enfermeiros. CIPE Versão 1.0: Classificação internacional para a prática de enfermagem. Tradução Heimar de Fátima Marin. São Paulo: ALGOL; 2007.

10. Tannure MC, Gonçalves AMP. SAE, sistematização da assistência de enfermagem: Guia prático. 2a ed. Rio de Janeiro: Guanabara Koogan; 2010.

11. Mattei FD, Toniolo FN, Malucelli A, Cubas MR. Uma visão da produção científica internacional sobre a classificação internacional para a prática de enfermagem. Rev. Gaúcha Enferm. 2011;32(4):823-31.

12. Nóbrega MML. Sistemas de classificação em enfermagem: um trabalho coletivo. Série Didática: Enfermagem no SUS. João Pessoa: Idéias; 2000.

13. Garcia TM, Nóbrega MML. Classificação internacional para a prática de enfermagem: inserção brasileira no projeto do conselho internacional de enfermeiras. Acta Paul. Enferm. 2009;22(n. esp):875-9.

14. Nóbrega MML, Garcia TR. Perspectivas de incorporação da classificação internacional para a prática de enfermagem (CIPE®) no Brasil. Rev Bras Enferm. 2005;58(2):227-30.

15. Nóbrega MML. Classificação internacional para a prática de enfermagem é projeto do CIE. Nursing. 2002;5(51):12-4.

16. Nóbrega MML, Garcia TR, Furtado LG, Albuquerque CC, Lima CLH. Terminologias de enfermagem: da taxonomia da NANDA à classificação internacional para a prática de enfermagem. Rev. enferm. UFPE on line. 2008;2(4):454-6.

17. Cubas MR, Silva SH, Rosso M. Classificação internacional para a prática de enfermagem (CIPE®): uma revisão de literatura. Rev. Eletr. Enf. [Internet] 2010;12(1):186-94. [acesso em 11 abr 2011] Disponível: http://www.fen.ufg. br/revista/v12/n1/v12n1a23.htm

18. Fernandes MGM, Nobrega MML, Garcia TR, MacedoCosta KNF. Análise conceitual: considerações metodológicas. Rev Bras Enferm. 2011;64(6):1150-6.
19. Ferreira A. Novo dicionário da língua portuguesa. Rio de Janeiro: Nova Fronteira; 1993.

20. OPAS: Organização Pan-Americana da Saúde [Internet]. Guia alimentar para crianças menores de 2 anos. [acesso em 12 abr 2011] Disponível: http://new.paho. org $/$ bra/index.php?option $=$ com_docman\&task $=$ doc details\&gid $=16 \&$ Itemid $=423$

21. Fortes H, Pacheco G. Dicionário médico. Rio de Janeiro. Editor Fábio M de Mello; 1973.

22. Furtado LG. Construção de uma nomenclatura de diagnósticos de enfermagem para a clínica médica do HULW/UFPB [dissertação]. João Pessoa (PB): Universidade Federal da Paraíba; 2007.

23. Organização Mundial da Saúde. The optimal duration of exclusive breastfeeding. Genova: OMS; 2002.

24. Ministério da Saúde (BR). Secretaria de Atenção à Saúde. Departamento de Atenção Básica. Saúde da criança: nutrição infantil: aleitamento materno e alimentação complementar. Brasília: Ministério da Saúde; 2009.

25. Santos CS, Coriolano MWL, Sette GCS, Lima LS. Alimentação complementar do lactente: subsídios para a consulta de enfermagem em puericultura. Cogitare enferm. [Internet] 2010;15(3) [acesso em 12 jan 2011]. Disponível: http://ojs.c3sl.ufpr.br/ojs2/index.php/cogitare/ article/view/18900/12208

26. Coca KP, Gamba MA, Silva RS, Abrão AC. Factors associated with nipple trauma in the maternity unit. J. Pediatr. 2009;85(4):341-5.

27. Wambach K, SH Campbell, Gill SL, Dodgson JE, Abiona TC, Heinig MJ. Clinical lactation practice: 20 years of evidence. J Hum Lact 2005;(21)3:245-58

28. Ministério da Saúde(BR). Agência Nacional de Vigilância Sanitária. Banco de leite humano: funcionamento, prevenção e controle de riscos. Brasília: Anvisa; 2008.

29. Rivemales MC, Azevedo ACC, Bastos PL. Revisão sistemática da produção científica da enfermagem sobre o desmame precoce. Rev. enferm. UERJ. 2010;18(1):132-7. 